How to reference this article Ivanovska-Naskova, R. (2019). Gli studi contrastivi dell'italianistica macedone: sviluppi e recenti prospettive. Italica Wratislaviensia, 10(1), 59-76.

DOI: http://dx.doi.org/10.15804/IW.2019.10.1.3

Ruska Ivanovska-Naskova

Univerzitet "Sv. Kiril i Metodij" - Skopje

rivanovska@flf.ukim.edu.mk, ORCID: 0000-0002-9819-6851

\title{
GLI STUDI CONTRASTIVI DELL'ITALIANISTICA MACEDONE: SVILUPPI RECENTI E PROSPETTIVE
}

\section{CONTRASTIVE ITALIAN-MACEDONIAN STUDIES: RECENT ADVANCES AND FUTURE PERSPECTIVES}

\begin{abstract}
The paper aims to present contrastive Italian-Macedonian studies, giving a general overview of the development of these studies, with particular attention to the context in which the first studies between these two languages appeared. A corpus of 60 studies published in the last two decades is analysed in the second part of the paper. The classification of the studies based on the topics covered reveals a prevalence of morphosyntactic, semantic, and translation studies. Contrastive studies related to teaching Italian as a foreign language and studies that introduce other topics are also present in the corpus. The final part of the paper reflects upon the future of this type of contrastive study, especially in light of the recent changes related to the interest in studying Italian in Macedonia.
\end{abstract}

Keywords: Italian, Macedonian, contrastive studies, translation studies, Italian as a foreign language 


\section{INTRODUZIONE}

T 'obiettivo del contributo è quello di esaminare il quadro degli studi _contrastivi italiano-macedone ${ }^{1}$. Il primo motivo per trattare questo argomento è legato al rinnovato interesse negli ultimi decenni per la linguistica contrastiva, che si deve soprattutto alle nuove prospettive metodologiche aperte dalla linguistica dei corpora. Il secondo motivo è da ricondurre ai legami linguistici, letterari e culturali italo-macedoni, che si sono intensificati in maniera particolare negli ultimi decenni attraverso lo sviluppo dell'italianistica macedone. Da qui nasce anche la necessità di fare una sistematizzazione degli studi contrastivi italiano-macedone e ragionare sulle prospettive future di ricerca.

La prima parte dello studio presenta una breve panoramica della storia dell'italianistica macedone, in cui nascono i primi studi di ordine contrastivo tra l'italiano e il macedone, e si esaminano i contesti in cui si studia l'italiano in Macedonia. Nella parte centrale, viene analizzato il corpus composto da circa sessanta studi pubblicati negli ultimi venti anni in base all'argomento trattato. Nella parte finale si riflette sulle possibili direzioni future degli studi contrastivi tra queste due lingue tenendo in conto anche i cambiamenti recenti nei contesti in cui si studia l'italiano in Macedonia.

\section{L'ITALIANISTICA MACEDONE}

I legami linguistici, letterari e culturali italo-macedoni, che trovano le proprie radici nei contatti tra le due culture in vari punti della storia dei Balcani $^{2}$, si sono intensificati grazie all'apertura del lettorato di lin-

1 L'articolo riprende una ricerca svolta nell'ambito del progetto Makedonskoromanistički jazični, kniževni i preveduvački relacii [Relazioni lingustiche, letterarie e traduttive tra il macedone e le lingue romanze] dell'Università "Ss. Cirillo e Metodio" di Skopje. La ricerca, presentata in parte nel contributo Kontrastivnite proučuvanja megju italijanskiot i makedonskiot jazik (2000-2015) (Ivanovska-Naskova, 2015), è stata approfondita ed estesa anche verso gli studi degli ultimi quattro anni.

2 Per una panoramica della storia dei legami italo-macedoni si veda Gjurčinova (2001, p. 47-56). 
gua italiana nel 1959 all'Università "Ss. Cirillo e Metodio" di Skopje (Poposki, 2001). Proprio in quell'anno, nel curriculum dell'allora Dipartimento di filologia romanza vengono inseriti per la prima volta gli studi biennali d'italiano. Da quel momento, l'italianistica in Macedonia è in continuo sviluppo: nel 1978 vengono introdotti gli studi triennali, nel 1997 il corso di laurea quadriennale di lingua e letteratura italiana e nel 2010 viene costituito il Dipartimento di lingua e letteratura italiana (Saržoska, 2011a, 2015a) .

Dall'intensificazione di questi legami italo-macedoni nascono i primi impulsi per studi contrastivi tra queste due lingue. Le poche ricerche di questo periodo sono focalizzate sulla trascrizione (Fonseca, 1991), sulle espressioni fraseologiche (Nikodinovska, R., 1997, 1999) e sugli italianismi nel macedone (Saržoska, 1993, 1997). Nel 1991, inoltre, nella rivista Literaturen zbor vengono presentate alcune ricerche di studenti dell'Università di Skopje, svolte sotto la guida del lettore Augusto Fonseca (Velkova, 1991; Miovska, 1991a; 1991b; Miševa, 1991; Sinadinovska, 1991a; 1991b; Fero, 1991).

Un altro fattore importante per il profilo degli studi contrastivi italiano-macedone è legato all'interesse per lo studio dell'italiano e ai contesti in cui si studia l'italiano in Macedonia (Saržoska, 2006; Jelen, 2013; Ivanovska-Naskova, 2017a; Saržoska \& Guido Srempf, 2017). Secondo i dati presentati nel portale Osservatorio del Ministero degli Affari Esteri e della Cooperazione Internazionale (Farnesina, n.d.), gli studenti che hanno studiato l'italiano in Macedonia nel biennio 2015/2016 sono 3.776. Quasi tre quarti degli studenti provengono da scuole locali (2826), mentre gli altri studiano l'italiano soprattutto all'università (662) o in altri contesti (288). La decisione del Governo macedone di introdurre l'italiano tra le lingue straniere che si studiano nell'arco di quattro anni di scuola elementare a partire dall'a.s. 2018/2019 apre la strada all'italiano nelle scuole pubbliche macedoni (Farnesina, 2018).

3 Un altro fattore importante, che ha favorito notevolmente lo scambio italo-macedone, è stata l'apertura del lettorato di lingua macedone presso l'Università degli Studi di Napoli l'Orientale nel 1969. Da questo centro, in quei tempi molto attivo e oggi, purtroppo in sospeso, si ricorda il volume La Fonetica del macedone (Minissi, Cinque \& Kitanovski, 1982). 


\section{LE RICERCHE CONTRASTIVE ITALIANO-MACEDONE DAL 2000 AD OGGI}

Il quadro degli studi contrastivi italiano-macedone segue lo sviluppo positivo dell'italianistica macedone. Il corpus analizzato comprende una sessantina di studi di undici autori che hanno affrontato vari argomenti. Si tratta di quattro studi monografici, una decina di articoli in riviste, quattro tesi di dottorato e tesi master e una quarantina di studi pubblicati in atti di convegni e miscellanee. La maggior parte delle ricerche è pubblicata in macedone, circa venti in italiano, una in francese e una, invece, in inglese. Molti degli autori sono docenti al Dipartimento di lingua e letteratura italiana a Skopje o si sono formati presso questa sede. Per quanto riguarda le tematiche affrontate, due studi sono dedicati alla trascrizione, una decina ad argomenti di ordine morfosintattico e altrettanti a tematiche strettamente sintattiche, le ricerche semantiche sono circa venti, mentre gli studi sul lessico in ottica contrastiva sei. Ci sono inoltre sei studi sui corpora, due di sociolinguistica, tre studi traduttologici e pochi studi che non rientrano nelle categorie citate. In seguito vengono presentati in linee generali le varie tipologie di studi, con attenzione particolare agli studi più corposi e agli autori rappresentati da un numero maggiore di ricerche.

Nel corpus di studi analizzati si riscontrano alcuni studi che vertono sulla trascrizione da una lingua all'altra. Questi argomenti sono condizionati dall'intensa attività traduttologica soprattutto nella direzione italiano - macedone (Gjurčinova, 2015). Il primo studio contrastivo, quello già citato di Fonseca (1991), è uno studio dedicato alla trascrizione in italiano di nomi slavi scritti in cirillico, tra cui anche quelli macedoni. Kitanovski (2001) esamina le regole da adottare nella trascrizione di nomi italiani in macedone e le non corrispondenze tra la grafia in cirillico e la pronuncia. Saržoska e Aleksoska (2015), invece, si soffermano sulla trascrizione dalla prospettiva didattica.

Nel sottocorpus di studi morfosintattici, i più numerosi sono quelli di Guido Srempf che esamina in modo sistematico gli errori presenti nell'interlingua di apprendenti macedoni dell'italiano. Il lavoro più significativo è la sua tesi master del 2003, Gli errori morfosintattici e les- 
sicali nell'apprendimento dell'italiano da parlanti macedoni (Guido Srempf, 2003a). Sulla stessa linea si situano altri suoi due studi (Guido Srempf, 2001, 2003b) e una sua ricerca del 2015 che verte sugli errori riscontrati nelle traduzioni studentesche e interpretati in chiave contrastiva (Guido Srempf, 2015). Anche due degli studi di Nikodinovska (2012b, 2015a) appartengono a questo sottogruppo: il primo è dedicato al verbo dovere e i suoi equivalenti in macedone, mentre l'altro si focalizza sulla traduzione di certe strutture grammaticali come possibile approccio nella didattica dell'italiano. Due altri studi sono dedicati al congiuntivo italiano e i suoi equivalenti nel macedone (Grivčevska 2011; Saržoska, Velevska \& Trajkova 2015). Uno studio di Nikodinovski (2014b) si occupa, invece, dei prefissi negativi nelle due lingue.

Le problematiche sintattiche in ottica contrastiva italiano-macedone, già presenti in alcuni studi citati precedentemente, si sviluppano in maniera più sistematica a partire dal 2011. Oltre alla tesi di dottorato di Ivanovska-Naskova, I costrutti condizionali nel corpus parallelo italiano macedone Italmac (Ivanovska-Naskova 2011a), si riscontrano altre due ricerche sulla forma e la semantica dei costrutti condizionali (Ivanovska-Naskova, 2013, 2014b). Le ricerche contrastive sui costrutti condizionali si estendono anche verso il serbo, con lo studio di Ivanovska-Naskova e Samardžić (2016) sulla codificazione della controfattualità nell'italiano, nel macedone e nel serbo. La ricerca di IvanovskaNaskova e Stojmenova (2015) verte, invece, sulla struttura valenziale di alcuni verbi nell'italiano e nel macedone, mentre altri tre studi si soffermano sulle preposizioni italiane, in particolare sugli errori commessi da apprendenti macedoni (Guido Srempf, 2013) e le funzioni sintattiche della preposizione $a$ e i suoi equivalenti nel macedone (Saržoska, 2011b, 2013b).

Nel corpus esaminato spiccano le ricerche semantiche sia per quanto concerne il numero di studi sia per la sistematicità con la quale vengono sviluppati certi argomenti nel corso degli anni. Di questo sottogruppo fanno parte innanzitutto le pubblicazioni di Nikodinovska, a partire dalla sua prima ricerca svolta nell'ambito dei suoi studi post lauream nel 2003 sulle espressioni fraseologiche nell'insegnamento dell'italiano ad apprendenti macedoni corredata da un glossario di espressioni fra- 
seologiche nelle due lingue (Nikodinovska, R., 2010a [2009]). Questa studiosa si è dedicata in più occasioni ad altri simili argomenti analizzati in prospettiva contrastiva come, per esempio, le metafore spaziali, i fraseologismi antroponimici, la flessibilità delle espressioni freseologiche, i fraseologismi avverbiali, i cromonimi e i toponimi nelle espressioni fraseologiche (Nikodinovska, R., 2000, 2001, 2002, 2003, 2004, 2010b, 2011a, 2011b, 2012a, 2015b, 2016b, 2018). Vanno menzionati in questo filone anche alcuni studi paremiologici. Il primo ad occuparsi dei proverbi italiani e macedoni è Veličkovski, che riflette sulla possibilità di utilizzare i proverbi nelle due lingue a scopi didattici (Veličkovski, 2001) e pubblica successivamente una raccolta di proverbi italiani e i loro equivalenti macedoni (Veličkovski, 2009). Altre ricerche si focalizzano su proverbi di un certo tipo nelle due lingue: proverbi con una parola chiave (Koceva, 2012), proverbi con un determinato contenuto semantico (Nikodinovska, D., 2011, 2013, 2015; Nikodinovski, 2014a) o proverbi con una determinata struttura sintattica (Conenna, Hadži-Lega Hristoska \& Ivanovska-Naskova, 2018). Due di questi studi concernono altre due lingue, l'articolo riguardante l'espressione del binomio il bene e il male è svolto anche su un corpus di proverbi giapponesi (Nikodinovska, D., 2015), quello sui proverbi con il se condizionale comprende anche esempi francesi (Conenna, Hadži-Lega Hristoska \& Ivanovska-Naskova, 2018).

Lo studio più approfondito sul lessico è la ricerca di Saržoska sugli italianismi nel macedone (Saržoska, 2009), a cui vanno aggiunti altri due studi su argomenti simili (Saržoska 2000, 2014) e un'altra sua ricerca sui neologismi nelle due lingue (Saržoska, 2015b). Le ricerche di Guido Srempf, come già accennato prima, vertono anche su queste tematiche. Accanto al suo principale studio sugli errori morfosintattici e lessicali di apprendenti macedoni (Guido Srempf, 2003a), ne troviamo anche un altro focalizzato sulle interferenze lessicali (Guido Srempf, 2007b).

Nel corpus si riscontrano anche studi che si occupano specificamente di corpora e delle prospettive che questi strumenti aprono nelle ricerche contrastive e nella didattica dell'italiano come lingua straniera. Il già citato lavoro di Ivanovska-Naskova sui costrutti condizionali (Iva- 
novska-Naskova, 2011a) è basato su un corpus parallelo di testi letterari specificamente compilato ai fini di questa ricerca. Altri suoi studi sono di natura metodologica e indagano sulla possibilità di utilizzare i corpora nelle ricerche contrastive e nella didattica dell'italiano LS (IvanovskaNaskova, 2011b, 2011c, 2011d, 2014a, 2018b).

Il denominatore comune della maggior parte degli studi citati è l'intenzione di utilizzarne i risultati nell'insegnamento dell'italiano ad apprendenti macedoni. Accanto agli studi già citati di Guido Srempf (2003a), Nikodinovska (2010a [2009]) e Ivanovska-Naskova (2011a) con una spiccata componente didattica, vanno menzionati altri due lavori di Guido Srempf(2007a, 2014). Il primo esamina i contenuti del corso di analisi contrastiva nell'ambito del Corso di laurea in Lingua e letteratura italiana presso l'Università di Skopje, mentre nell'altro si propongono materiali didattici concreti basati sulla traduzione di pubblicità italiane e analisi di testi paralleli in classe. Particolarmente significativo in questa prospettiva didattica è lo studio di Nikodinovska (2009), La didattica e la valutazione della traduzione dall'italiano in macedone. Uno studio recente di Hadži-Lega Hristoska e Ivanovska-Naskova (2017) indaga la possibilità di utilizzare risorse elettroniche nell'insegnamento della fonologia francese e italiana ad apprendenti macedoni partendo anche da alcune osservazioni contrastive che riguardano queste due lingue romanze e il macedone.

Alla fine di questo elenco, vanno menzionati anche alcuni studi che non appartengono a nessuno dei filoni presentati: uno studio pragmatico (Rodrigez, 2002), alcuni studi traduttologici (Nikodinovska, R., 2014a, 2014b, 2016a, 2016c, 2017; Grivčevska, 2015), due studi sociolinguisici, uno di Saržoska (2013a) su titoli di giornali, e l'altro di Stojmenova Weber (Stojmenova, 2015) sul fenomeno del code-switching in famiglie macedoni residenti nella Svizzera di lingua italiana, e il più recente studio di Stojmenova Weber sulla punteggiatura nelle due lingue (Stojmenova Weber, 2019).

Va menzionato alla fine un manuale pubblicato recentemente, Вовед во граматиката на италијанскиот јазик [Introduzione alla grammatica italiana] destinato agli studenti del primo anno all'Università "Ss. Cirillo e Metodio" di Skopje in cui sono introdotte delle osservazio- 
ni contrastive (Ivanovska-Naskova, 2018a). Gli studenti, attraverso le attività proposte, sono invitati a riflettere paragonando le due lingue su alcuni argomenti, come per esempio, le differenze nei sistemi fonologici, le differenze che riguardano la forma e le funzioni dell'articolo nelle due lingue, le somiglianze nei sistemi dei suffissi alterativi ecc. Lo scopo di queste attività è stimolare la riflessione metalinguistica in chiave contrastiva che contribuisce non solo alle conoscenze strettamente linguistiche degli studenti, ma anche allo sviluppo delle loro abilità critiche.

\section{CONCLUSIONI}

Il quadro appena tracciato mostra come lo sviluppo degli studi contrastivi italiano-macedone segua la traiettoria positiva dell'italianistica macedone. Le ricerche affrontano un ampio ventaglio di argomenti, tra cui spiccano le tematiche morfosintattiche e semantiche. In alcuni domini, e in modo particolare, negli studi semantici, si nota una continuità nel portare avanti questo tipo di ricerche. Con poche eccezioni, quasi tutte le ricerche sono didatticamente motivate e presentano attività concrete da proporre in classe. Alcuni studi riportano anche le esperienze di queste sperimentazioni che, in particolar modo quando si avvalgono di strumenti come dizionari elettronici o corpora, risultano particolarmente stimolanti. Gli studi sul lessico, inoltre, trovano impiego nella lessicogafia, in particolare nella stesura di dizionari bilingui. Le applicazioni di queste ricerche contrastive sono una prova delle loro potenzialità da sfruttare. La presenza di studi sui corpora o argomenti come il code switching sono anche una prova del fatto che gli studi contrastivi italiano-macedone sono in linea con alcuni degli argomenti attuali delle ricerche linguistiche e che il quadro appena tracciato si arricchisce continuamente non solo per quanto riguarda il numero di studi contrastivi, ma anche per gli argomenti affrontati. La dinamica con cui si sono sviluppati questi studi negli ultimi due decenni promette un ulteriore arricchimento del quadro. Per quanto riguarda le tematiche, si auspicano in futuro anche studi su argomenti meno sviluppati, come per esempio, i sistemi verbali o la testualità. Si spera inoltre che la recente introduzione dell'italiano nel sistema scolastico macedone come seconda lingua straniera possa 
aprire gli studi contrastivi italiano-macedone anche a tematiche focalizzate sull'interlingua di apprendenti di età tra 10 e 18 anni che imparano l'italiano in un contesto formale.

\section{BIBLIOGRAFIA}

Farnesina (2018, April 09). Il governo introduce la lingua italiana nel sistema scolastico macedone. Retrieved from https://ambskopje.esteri.it/ambasciata_skopje/it/ambasciata/news/eventi-culturali/2018/04/il-governointroduce-la-lingua.html.

Farnesina (n.d.). Osservatorio. "Li" Il portale della lingua italiana. Retrieved from https://www.linguaitaliana.esteri.it/lingua/osservatorio/dettaglio. do.

Gjurčinova, A. (2001). Italijanskata kniževnost vo Makedonija. Skopje: Institut za makedonska literatura.

Gjurčinova, A. (2015). La letteratura italiana del Novecento e le sue traduzioni in lingua macedone. In V. Zaccaro \& R. Ivanovska-Naskova (Eds.), Incroci. Studi sulla letteratura, la traduzione, la glottodidattica (pp. 67-76). Bari/Skopje: Università degli Studi di Bari Aldo Moro/Università "Ss. Cirillo e Metodio" di Skopje.

Ivanovska-Naskova, R. (2017a). Studiare l'italiano in Macedonia. In P. Guaragnella, R. Abbaticchio \& C. Williams (Eds.), Diffusione della lingua italiana e ruolo dei Centri Linguistici di Ateneo (pp. 43-52). Lecce: Pensa Multimedia.

Ivanovska-Naskova, R. (2015). Kontrastivnite proučuvanja megju italijanskiot i makedonskiot jazik (2000-2015). In I. Babamova (Ed.), Makedonskoromanistički jazični, kniževni i preveduvački relacii (pp. 211-228). Skopje: Univerzitet "Sv. Kiril i Metodij”.

Jelen, A.K. (2013). Il boom dell' 'italiano in Macedonia. In.it., 29-30, 53-56.

Minissi, N., Kitanovski, N., \& Cinque, U. (1982). La fonetica del macedone. Napoli: Bibliopolis.

Poposki, A. (2001). Italijanskiot jazik na Filološkiot fakultet "Blaže Koneski”. In P. Atanasov (Ed.), Atti del Convegno 40 anni di lettorato di italiano all'Università "Ss. Cirillo e Metodio" - Skopje (10-11 novembre 2000, Skopje) (pp. 28-35). Skopje: Università "Ss. Cirillo e Metodio" di Skopje. 
Saržoska, A. (2006). Macedonia - l'italiano scelto dai giovani come investimento per il futuro. In P. Peluffo \& L. Serianni (Eds.), Il mondo in italiano. Annuario 2006 della Società Dante Alighieri (pp. 271-285). Roma: DA.

Saržoska, A. (2011a). 50 anni di studi italiani alla Facoltà di Filologia "Blaže Koneski” - Skopje. In R. Nikodinovska (Ed.), Lingua, letteratura e cultura italiana. Atti del Convegno internazionale (pp. 17-25). Skopje: Università "Ss. Cirillo e Metodio" di Skopje.

Saržoska, A. (2015a). L'Italianistica in Macedonia: lo stato dell'arte. In V. Zaccaro \& R. Ivanovska-Naskova (Eds.), Incroci. Studi sulla letteratura, la traduzione, la glottodidattica (pp. 153-162). Bari/Skopje: Università degli Studi di Bari Aldo Moro/Università "Ss. Cirillo e Metodio" di Skopje.

Saržoska A., \& Guido Srempf, L. (2017). L'insegnamento dell'italiano in Macedonia. In P. Diadori \& E. Carrea (Eds.), La NUOVA DITALS risponde (pp. 391-394). Roma: Edilingua.

\section{Corpus di studi analizzati}

Conenna, M., Hadži-Lega Hristoska, J., \& Ivanovska-Naskova, R. (2018). Comparaison des proverbes français, italiens et macédoniens avec les connecteurs conditionnels si/selako. In Z. Nikodnovski (Ed.), Le même, le semblable et le différent au sein de la langue, de la littérature et de la culture dans les pays francophones (pp. 124-146). Skopje: Université "Sts Cyrille et Méthode".

Fero, A. (1991). Nekoi formi so imperativno značenje vo italijanskite korisni soveti i nivniot makedonski ekvivalent. Literaturen zbor, 38(1/2), $167-175$.

Fonseca, A. (1991). Trascrizione in lingua italiana di parole appartenenti alle lingue slave che usano l'alfabeto cirillico. Rassegna italiana di Linguistica Applicata, XXIII(2), 93-113.

Guido Srempf, L. (2001). Alcuni degli errori più comuni commessi dagli studenti macedoni nell'apprendimento dell'italiano. In P. Atanasov (Ed.), Atti del Convegno 40 anni di lettorato di italiano all'Università "Ss. Cirillo e Metodio" - Skopje (10-11 novembre 2000, Skopje) (pp. 123-134). Skopje: Università "Ss. Cirillo e Metodio".

Guido Srempf, L. (2003a). Morfosintaksički i leksički jazični greski vo izučuvanjeto na italijanskiot jazik od makedonski govoriteli [Master's thesis]. Skopje: Univerzitet "Sv. Kiril i Metodij”. 
Guido Srempf, L. (2003b). Morfosintaksički i leksički jazični greski vo izučuvanjeto na italijanskiot jazik od makedonski govoriteli. Godišen zbornik, 28/29, 153-159.

Guido Srempf, L. (2007a). L'insegnamento dell'analisi contrastiva presso il Dipartimento d'italianistica dell'Università "Ss. Cirillo e Metodio" di Skopje. In A. Gjurčinova \& V. Zaccaro (Eds.), Tempo d' incontri. Atti dei seminari TEMPUS (JEP 18101-2003) (pp. 26-34). Skopje: Università "Ss. Cirillo e Metodio".

Guido Srempf, L. (2007b). Interferenze lessicali nella traduzione: macedone ed italiano a confronto". In A. Gjurčinova \& V. Zaccaro (Eds.), Tempo d'incontri. Atti dei seminari TEMPUS (JEP 18101-2003) (pp. 247-257). Skopje: Università "Ss. Cirillo e Metodio".

Guido Srempf, L. (2013). Gli errori più frequenti commessi dai discenti macedoni nell'uso delle preposizioni italiane. In J. Vučo \& O. Durbaba (Eds.), Language and Education (pp. 87-103). Belgrade: University of Belgrade.

Guido Srempf, L. (2014). Analisi linguistica e traduzione della pubblicità macedone in una classe di italiano L2: una proposta didattica. In M. Aleksoska-Čkatroska \& J. Hadži-Lega Hristoska (Eds.), Romanistika i balkanistika. Zbornik na trudovi vo čest na prof. d-r Petar Atanasov po povod 75 godini od zivotot (pp. 269-287). Skopje: Univerzitet „Sv. Kiril i Metodij”.

Guido Srempf, L. (2015). Su alcuni errori interlinguistici in studenti macedoni di Italiano L2 nelle traduzioni L1/L2. In V. Zaccaro \& R. IvanovskaNaskova (Eds.), Incroci. Studi sulla letteratura, la traduzione, la glottodidattica (pp. 87-103). Bari/Skopje: Università degli Studi di Bari Aldo Moro/Università "Ss. Cirillo e Metodio" di Skopje.

Grivčevska, B. (2011). Il congiuntivo nell'italiano e i suoi corrispondenti nel macedone. In R. Nikodinovska (Ed.), Atti del Convegno internazionale "Lingua, letteratura e cultura italiana - 50 anni di Studi italiani" (29-30 ottobre 2009) (pp. 377-386). Skopje: Università „Ss. Cirillo e Metodio".

Grivčevska, B. (2015). Analisi degli errori commessi dagli studenti macedoni in classe di interpretazione simultanea. In R. Nikodinovska (Ed.). Parallelismi linguistici, letterari e culturali - 55 anni di Studi italiani all'Università “Ss. Cirillo e Metodio” di Skopje (pp. 275-294). Skopje: Università "Ss. Cirillo e Metodio". 
Hadži-Lega Hristoska, J., \& Ivanovska-Naskova, R. (2017). Nastavata po fonetika $\mathrm{i}$ fonologija na francuskiot i na italijanskiot jazik pomegju tradicijata i inovacijata. In S. Petrova (Ed.), Jazik, literatura i kultura vo romanskiot prostor: tradicija i inovacija (pp. 213-228). Skopje: Univerzitet „Sv. Kiril i Metodij”.

Ivanovska-Naskova, R. (2011a). I costrutti condizionali nel corpus parallelo italiano-macedone Italmac [Doctoral dissertation]. Bari: Università degli Studi di Bari Aldo Moro.

Ivanovska-Naskova, R. (2011b). Italian-Macedonian parallel corpus. In M. Konopka, J. Kubczak, C. Mair, F. Štícha \& U.H. Njaßner (Eds.), Grammatik und Korpora 2009 (pp. 599-604). Tübingen: Narr Verlag.

Ivanovska-Naskova, R. (2011c). I corpora paralleli come fonti di esempi per analisi contrastiva: Italmac e ricerche contrastive tra l'italiano e il macedone. La Nuova Ricerca, 20, 287-296.

Ivanovska-Naskova, R. (2011d). Progettazione e costruzione di un corpus parallelo italiano-macedone. In R. Nikodinovska (Ed.), Lingua, letteratura e cultura italiana. Atti del Convegno internazionale (pp. 219-238). Skopje: Università "Ss. Cirillo e Metodio".

Ivanovska-Naskova, R. (2013). I costrutti formalmente condizionali nell'italiano e nel macedone. Italica Belgradensia, 2/2013, 171-181.

Ivanovska-Naskova, R. (2014a). Il corpus parallelo italiano-macedone come strumento nella didattica dell'italiano LS. In E. Garavelli \& E. SuomelaHärmä (Eds.), Dal manoscritto al web: canali e modalità di trasmissione dell'italiano. Tecniche, materiali e usi nella storia della lingua. Atti del XII Congresso SILFI Società Internazionale di Linguistica e Filologia Italiana (Helsinki, 18-20 giugno 2012) (pp. 471-479). Firenze: Franco Cesati.

Ivanovska-Naskova, R. (2014b). I connettivi condizionali nell'italiano e nel macedone: analisi contrastiva attraverso un corpus parallelo. In P. Macaluso (Ed.), La lingua variabile nei testi letterari, artistici e funzionali contemporanei. Analisi, interpretazione, traduzione. Testi presentati al XIII Congresso della SILFI Società Internazionale di Linguistica e Filologia Italiana (Palermo, 22-24 settembre 2014) [CD]. Palermo: Centro di studi filologici e linguistici siciliani.

Ivanovska-Naskova, R. (2018a). Voved vo gramatikata na italijanskiot jazik. Skopje: Univerzitet "Sv. Kiril i Metodij". Retrived from http://www. ukim.edu.mk/e-izdanija/FLF/Voved_vo_gramatikata_na_italijanskiot_ jazik_2018.pdf. 
Ivanovska-Naskova, R. (2018b). L'insegnamento della grammatica dell'italiano LS attraverso corpora. Italica Wratislaviensia, 9(1), 71-87.

Ivanovska-Naskova, R., \& Samardžić, M. (2016). La codificazione della controfattualità nell'italiano, nel macedone e nel serbo. In O. Inkova \& A. Trovesi (Eds.), Langues slaves en contraste / Славянские языки in comparatione / Lingue slave a confronto (pp. 85-107). Bergamo: Bergamo University Press.

Ivanovska-Naskova, R., \& Stojmenova, R. (2015). La struttura valenziale di alcuni verbi di alta frequenza nell'italiano e nel macedone. In A. Klimkiewicz, M. Malinowska, A. Paleta \& M. Wrana (Eds.), L'Italia e la cultura europea (pp. 567-576). Firenze: Franco Cesati.

Kitanovski, N. (2001). Otstapuvanje od izgovorot i transkripcijata na italijanskite iminja vo segašniot pravopis na makedonskiot literaturen jazik. Literaturen zbor, 48 (3/4), 33-37.

Koceva, V. (2012). Semiologijata na gjavolot vo makedonskiot i vo italijanskiot jazik, Literaturen zbor, 60(1-3), 93-101.

Miovska, S. (1991a). Za eden vid italijanski skratenici i nivnata lekcikalizacija (so nekoi paraleli vo makedonskiot jazik). Literaturen zbor, 38(1/2), 160-166.

Miovska, S. (1991b). Za frazeologizmite vo italijanskiot i makedonskiot jazik. Literaturen zbor, 38(1/2), 150-153.

Miševa, M. (1991). Glagolskite formi na prostiot i složeniot kondicional vo italijanski jazik i nivnite možni soodvetstva vo makedonskiot. Literaturen zbor, 38(1/2), 179-183.

Nikodinovska, D. (2011). Vrednosnata oska dobro/loso vo italijanskite i vo makedonskite poslovici. Godišen zbornik na Filološkiot fakultet na Univerzitetot „Goce Delčev” vo Štip, 2, 61-71.

Nikodinovska, D. (2013). Aksiološkata oska ubavo/grdo vo poslovicite vo italijanskiot i vo makedonskiot jazik. In J. Vučo \& V. Polovina (Eds.), Savremeni tokovi u lingvističkim istraživanjima. Knjiga 2 (pp. 75-90). Beograd: Univerzitet u Beogradu.

Nikodinovska, D. (2015). Dobroto $i$ lošoto kako vrednosni kategorii vo poslovicite vo italijanskiot, vo japonskiot $i$ vo makedonskiot jazik [Doctoral dissertation]. Skopje: Univerzitet "Sv. Kiril i Metodij".

Nikodinovska, R. (1997). Kvantitativni frazeološki izrazi vo italijanskiot i makedonskiot jazik. Godišen zbornik na Filološkiot fakultet "Blaže Koneski” na Univerzitetot "Sv. Kiril i Metodij”, 23, 169-173. 
Nikodinovska, R. (1999). Kvantitativni frazeološki izrazi vo italijanskiot i makedonskiot jazik. In G.J., Lumburovski, K. Dobruševski \& T. Ognenovski (Eds.), Makedonsko-italijanski odnosi. Zbornik na trudovi podneseni na naučniot sobir odrzan na 10-11 maj 1996 god. (pp. 273-276). Bitola: Društvo za nauka i umetnost.

Nikodinovska, R. (2000). Sporedbeni italijansko-makedonski frazeološki proučuvanja. In XXVI Naučna konferencija na XXXII Megjunaroden seminar za makedonski jazik, literatura i kultura, Ohrid (pp. 303-314). Skopje: Univerzitet "Sv. Kiril i Metodij”.

Nikodinovska, R. (2001). Prostorni metafori vo italijanskiot i vo makedonskiot jazik. In XXVII Naučna konferencija na XXXIII Megjunaroden seminar za makedonski jazik, literatura i kultura, Ohrid, 14-15 avgust 2000 (pp. 177-185). Skopje: Univerzitet “Sv. Kiril i Metodij”.

Nikodinovska, R. (2002). Antroponimiski frazemi vo italijanskiot i vo makedonskiot jazik. XXVIII Naučna konferencija na XXXIV Megjunaroden seminar za makedonski jazik, literatura i kultura, Ohrid, 13-14 avgust 2001 (pp. 203-218). Skopje: Univerzitet “Sv. Kiril i Metodij”.

Nikodinovska, R. (2003). Vizuelni metafori vo italijanskiot i vo makedonskiot jazik. XXIX Naučna konferencija na XXXV Megjunaroden seminar za makedonski jazik, literatura i kultura, Ohrid, 19-20 avgust 2002 (pp. 483-490). Skopje: Univerzitet “Sv. Kiril i Metodij”.

Nikodinovska, R. (2004). Fleksibilnosta na frazeološkite izrazi vo italijanskiot i vo makedonskiot jazik. XXX Naučna konferencija na XXXVI Megjunaroden seminar za makedonski jazik, literatura i kultura, Ohrid, 11-13 avgust 2003 (pp. 391-400). Skopje: Univerzitet "Sv. Kiril i Metodij".

Nikodinovska, R. (2009). Didaktika i evaluacija na preveduvanjeto od italijanski na makedonski jazik i obratno. Skopje: Univerzitet "Sv. Kiril i Metodij".

Nikodinovska, R. (2010a [2009]). Nastava na italijanskata frazeologija. Skopje: Univerzitet "Sv. Kiril i Metodij”.

Nikodinovska, R. (2010b). Paralelni proučuvanja na frazemite. Godišen zbornik na Filološkiot fakultet „Blaže Koneski” na Univerzitetot “Sv. Kiril i Metodij”, 36, 235-261.

Nikodinovska, R. (2011a). Il colore 'nero' nelle espressioni fraseologiche italiane e macedoni. In Un secol de italienistică la Bucureşti (pp. 495-501). Bucureşti: Editura Universităţii Bucureşti. 
Nikodinovska, R. (2011b). Adverbijalni frazemi vo italijanskiot i vo makedonskiot jazik. In XXXVII Naučna konferencija na XLIII Megjunaroden seminar za makedonski jazik, literatura i kultura, Ohrid, 15-16 juli 2010 (pp. 233-241). Skopje: Univerzitet "Sv. Kiril i Metodij”.

Nikodinovska, R. (2012a). I colori nelle espressioni fraseologiche italiane, slovene e macedoni. In A. Žabjek \& L.G. Scuteri (Eds.), I 90 anni dello sloveno a Napoli (pp. 109-116). Napoli: Università degli studi di Napoli L'Orientale.

Nikodinovska, R. (2012b). Glagolot dovere i negovite ekvivalenti vo makedonskiot jazik. In XXXVIII Naučna konferencija na XLIV Megjunaroden seminar za makedonski jazik, literatura i kultura, Ohrid, 14-15 juli 2011 (pp. 141-147). Skopje: Univerzitet “Sv. Kiril i Metodij”.

Nikodinovska, R. (2014a). Preveduvackite strategii vo makedonskiot prevod na dramata Henri IV od Pirandelo. In XL Naučna konferencija na XLVI Megjunaroden seminar za makedonski jazik, literatura i kultura, Ohrid, 29-30 juni 2013 (pp. 645-657). Skopje: Univerzitet "Sv. Kiril i Metodij".

Nikodinovska, R. (2014b). Interkulturen transfer vo preveduvanjeto niz primeri od makedonskiot prevod na romanot Pepel od Gracija Deleda. In M. Aleksoska-Čkatroska \& J. Hadži-Lega Hristoska (Eds.), Romanistika i balkanistika. Zbornik na trudovi vo čest na prof. d-r Petar Atanasov po povod 75 godini od zivotot (pp. 539-545). Skopje: Univerzitet "Sv. Kiril i Metodij".

Nikodinovska, R. (2015a). La didattica della lingua italiana a studenti macedoni attraverso la traduzione di contenuti grammaticali. In V. Zaccaro \& R. Ivanovska-Naskova (Eds.), Incroci. Studi sulla letteratura, la traduzione, la glottodidattica (pp. 143-151). Bari/Skopje: Università degli Studi di Bari Aldo Moro, Università "Ss. Cirillo e Metodio" di Skopje.

Nikodinovska, R. (2015b). Hromonimite crveno, zolto i zeleno vo italijanskite i vo makedonskite frazeološki izrazi. In XLI Megjunarodna naučna konferencija na XLVII Megjunaroden seminar za makedonski jazik, literatura i kultura, Ohrid, 14-15 juni 2014 (pp. 85-100). Skopje: Univerzitet "Sv. Kiril i Metodij".

Nikodinovska, R. (2015-2016a). Kulturospecifičnite elementi vo makedonskiot prevod na romanot Eden, nieden i stoiljadi od Luidži Pirandelo, Godišen zbornik na Filološkiot fakultet "Blaže Koneski" na Univerzitetot "Sv. Kiril i Metodij" vo Skopje, 41/42, 339-349. 
Nikodinovska, R. (2016b). Sporedbena analiza na italijanski i makedonski frazemi od religioznata sfera so komponenta Gospod i Gjavol. In B. Mirčevska-Boševa (Ed.), Zborot zbor otvora (pp. 229-243). Skopje: Univerzitet "Sv. Kiril i Metodij”.

Nikodinovska, R. (2016c). Onomastikata vo makedonskiot prevod na Oslobodeniot Erusalim. In XLII Megjunarodna naučna konferencija na XLVIII Megjunaroden seminar za makedonski jazik, literatura i kultura (Ohrid, 13-27 juni 2015) (pp. 141-154). Skopje: Univerzitet "Sv. Kiril i Metodij".

Nikodinovska, R. (2017). Za kategorijata „neprevodlivoto vo prevodot”. In XLIII megjunarodna naučna konferencija na XLIX megjunaroden seminar za makedonski jazik, literatura i kultura Ohrid, (25-26 juni 2016) (pp. 40-49). Skopje: Univerzitet "Sv. Kiril i Metodij".

Nikodinovska, R. (2018). Toponimite vo italijanskite i vo makedonskite frazemi: sličnosti i razliki. In K. Veljanovska \& B. Mirčevska-Boševa (Eds.), Iminjata i frazeologijata (pp. 221-232). Skopje: Univerzitet "Sv. Kiril i Metodij".

Nikodinovski, Z. (2014a). I parallelismi nella fraseologia e paremiologia italiana e macedone. In M. Aleksoska-Čkatroska \& J. Hadži-Lega Hristoska (Eds.), Romanistika i balkanistika. Zbornik na trudovi vo čest na prof. d-r Petar Atanasov po povod 75 godini od životot (pp. 547-567). Skopje: Univerzitet "Sv. Kiril i Metodij”.

Nikodinovski, Z. (2014b). I prefissi verbali negativi in italiano e in macedone. In R. Nikodinovska (Ed.), Parallelismi linguistici, letterari e culturali55 anni di Studi italiani all' Università "Ss. Cirillo e Metodio" di Skopje (pp. 435-444). Skopje: Università "Ss. Cirillo e Metodio".

Rodrigez, V. (2002). Indirekten nacin za izrazuvanje na baranje: italijansko-makedonski sporedbi. In XXVIII Naučna konferencija na XXXIV Megjunaroden seminar za makedonski jazik, literatura i kultura, Ohrid, 13-14 avgust 2001 (pp. 345-352). Skopje: Univerzitet "Sv. Kiril i Metodij".

Saržoska, A. (1993). Italijanizmite vo makedonskiot jazik (od sferata na: bankarstvoto, modata, voenata i pomorskata terminologija). In Prv naučen sobir na mladi makedonisti (20-21 maj 1993) (pp. 267-280). Skopje: Univerzitet "Sv. Kiril i Metodij".

Saržoska, A. (1997). Zaemkite od italijansko poteklo vo makedonskiot jazik [Master's thesis]. Skopje: Univerzitet "Sv. Kiril i Metodij”. 
Saržoska, A. (2000). Za nekoi italijanski zaemki od domenot na oblekuvanjeto vo makedonskiot jazik. In P. Atanasov (Ed.), Prvi romanističko-balkanistički sredbi - vo čest na Božidar Nastev (pp. 219-226). Skopje: Univerzitet "Sv. Kiril i Metodij".

Saržoska, A. (2009). Italijanizmite vo makedonskiot jazik. Skopje: Univerzitet "Sv. Kiril i Metodij".

Saržoska, A. (2011b). Za predlogot A vo italijanskiot jazik i negovite možni ekvivalenti vo makedonskiot jazik. In XXXVII Naučna konferencija na XLIII Megjunaroden seminar za makedonski jazik, literatura i kultura, Ohrid, 15-16 juli 2010 (pp. 115-124). Skopje: Univerzitet "Sv. Kiril i Metodij”.

Saržoska, A. (2013a). Nekoi osobenosti na naslovite vo dnevniot pecat vo italijanskiot i vo makedonskiot jazik. In XXXIX Naučna konferencija na XLV Megjunaroden seminar za makedonski jazik, literatura i kultura, Ohrid, 20-21 juni 2012 (pp. 71-81). Skopje: Univerzitet “Sv. Kiril i Metodij”.

Saržoska, A. (2013b). Proposte di insegnamento/apprendimento della preposizione italiana A agli studenti macedoni. In P. Bianchi De Vecchi, A. Banjević \& M. Krivokapić Knežević (Eds.), New Approaches to Foreign Languages Didactics (pp. 91-97). Perugia: Guerra.

Saržoska, A. (2014). Gli italianismi nella lingua macedone: la nozione del prestito e i termini che la designano. In M. Aleksoska-Čkatroska \& J. Hadži-Lega Hristoska (Eds.), Romanistika i balkanistika. Zbornik na trudovi vo čest na prof. d-r Petar Atanasov po povod 75 godini od životot (pp. 643-649). Skopje: Univerzitet "Sv. Kiril i Metodij”.

Saržoska, A. (2015b). Neologizmite vo modernite sredstva za komunikacija vo italijanskiot i vo makedonskiot jazik: sličnosti i razliki. In I. Babamova (Ed.), Makedonsko-romanistički jazični, kniževni i preveduvački relacii (pp. 229-239). Skopje: Univerzitet "Sv. Kiril i Metodij".

Saržoska, A., \& Aleksoska, G. (2015). Transkripcija na italijanskite iminja vo makedonskiot jazik. In R. Nikodinovska (Ed.), Parallelismi linguistici, letterari e culturali - 55 anni di Studi italiani all'Università "Ss. Cirillo e Metodio" di Skopje (pp. 503-511). Skopje: Università "Ss. Cirillo e Metodio".

Saržoska, A.,Velevska, M., \& Trajkova, M. (2015). Subjunktivot vo italijanskiot, vo francuskiot i vo makedonskiot jazik, sličnosti i razliki. In R. Nikodinovska (Ed.), Parallelismi linguistici, letterari e culturali 55 anni di Studi italiani all'Università "Ss. Cirillo e Metodio" di Skopje (pp. 543-552). Skopje: Università "Ss. Cirillo e Metodio". 
Sinadinovska, J. (1991a). Italijanskite zborovi vo reklamnite natpisi vo Skopje. Literaturen zbor, 38(1/2), 142-146.

Sinadinovska, J. (1991b). Obid za sporeduvanje na formite za obrakanje vo makedonskiot i italijanskiot jazik. Literaturen zbor, 38(1/2), 153-160.

Stojmenova, R. (2015). Commutazione di codice italiano-macedone in Svizzera. In R. Nikodinovska (Ed.), Parallelismi linguistici, letterari e culturali - 55 anni di Studi italiani all'Università "Ss. Cirillo e Metodio” di Skopje (pp. 521-531). Skopje: Università "Ss. Cirillo e Metodio".

Stojmenova Weber, R. (2019). Tradurre la lineetta singola: uno studio contrastivo macedone-italiano. Italica Wratislaviensia, 10(1), XX.

Veličkovski, B. (2001). Makedonsko-italijanski paraleli vo poslovicite i možnosta za nivnoto vklučuvanje vo obrazovniot proces. In P. Atanasov (Ed.), Atti del Convegno 40 anni di lettorato di italiano all'Università "Ss. Cirillo e Metodio" - Skopje (10-11 novembre 2000, Skopje) (pp. 208-225). Skopje: Università "Ss. Cirillo e Metodio".

Veličkovski, B. (2009). Italijanski poslovici i pogovorki i nivnite makedonski ekvivalenti. Skopje: Institut za folklor "Marko Cepenkov".

Velkova, J. (1991). Za značenjeto na prostata i složenata forma na idnoto vreme vo italijanskiot jazik i nivnite možni soodvetstva vo makedonskiot. Literaturen zbor, 38(1/2), 175-179.

\footnotetext{
Riassunto: Lo studio mira a presentare il quadro delle ricerche contrastive italiano-macedone. Si presenta una breve panoramica della storia dell'italianistica macedone, con attenzione particolare al contesto in cui nascono i primi studi di ordine contrastivo tra l'italiano e il macedone. Nella parte centrale del contributo viene analizzato il corpus composto da circa sessanta studi pubblicati negli ultimi due decenni. La classificazione degli studi esaminati in base all'argomento trattato rivela la prevalenza di studi che vertono su argomenti morfosintattici, semantici e traduttologici. Negli ultimi anni si attesta inoltre una notevole crescita degli studi didatticamente motivati e ricerche che aprono argomenti nuovi. Nella parte finale dello studio si riflette sulle possibili direzioni future degli studi contrastivi tra queste due lingue tenendo in conto anche i cambiamenti recenti che riguardano l'interesse per l'italiano in Macedonia.
}

Parole chiave: italiano, macedone, studi contrastivi, studi traduttologici, italiano LS 